\title{
Recruiting CD33 on mast cells to inhibit IgE-mediated mast cell-dependent anaphylaxis
}

\author{
Stephen J. Galli \\ Departments of Pathology and Microbiology and Immunology, Stanford University School of Medicine, Stanford, California, USA.
}

\begin{abstract}
IgE-mediated activation of mast cells is a hallmark of an anaphylactic reaction to allergen. In this issue of the $J C I$, Duan et al. describe an approach for suppressing IgE-dependent mast cell activation, thereby suppressing anaphylaxis. Specifically, the authors show that delivery of liposomes containing both the specific antigen recognized by the mast cell-bound IgE and a high-affinity glycan ligand of the inhibitory receptor CD33 (CD33L) to targeted mast cells inhibits antigen-induced, FcERI-dependent spleen tyrosine kinase (Syk) phosphorylation and downstream protein tyrosine kinase (PTK) phosphorylation, $\mathrm{Ca}^{++}$flux, and $\beta$-hexosaminidase release (i.e., degranulation). However, this strategy only worked if both the antigen (reactive with the mast cell-bound IgE) and CD33L were on the same liposome. This approach promises to rapidly reduce IgE-dependent mast cell activation in response to challenge with offending allergens.
\end{abstract}

\section{Challenges with preventing or reversing fatal allergic reactions}

The ability to rapidly reverse potentially fatal IgE-dependent immunological reactivity to otherwise innocuous antigens, such as those present in foods, has become an area of increasing theoretical and practical importance. Such reactions are considered examples of acute anaphylaxis, which can manifest clinically within a few minutes of exposure of appropriately sensitized patients to even tiny amounts of the offending antigen, such as small amounts of peanut allergen contaminating an otherwise innocuous food (1). Despite much effort, the only effective approach currently available for interfering with such severe reactions once they have been triggered is the administration of epinephrine, typically via intramuscular injection (2). This approach requires minimization of the response time to be effective, as the epinephrine injection must be delivered with- in a few minutes of exposure of the patient to the offending allergen (2). In severe cases, delays of even a few minutes, such as might occur because of failure to make the correct diagnosis or failure to have ready access to epinephrine, can prove fatal (3).

Several groups have attempted to safely desensitize the major effector cell of anaphylaxis, the mast cell, by slowly increasing the amounts of allergen administered to the patient in a process termed allergen immunotherapy $(4,5)$. This approach requires the patient to be both correctly diagnosed as having anaphylactic reactivity to the offending allergen and willing to undergo months of allergen immunotherapy; however, this strategy is not without risks, including inadvertent elicitation of anaphylaxis that requires treatment with epinephrine. Thus, while conventional allergen immunotherapy can, in a best-case scenario, result in a patient being able to tolerate the accidental ingestion of a relatively high concen-

Related Article: p. 1387

Conflict of interest: SJG is a limited partner in KdT Ventures Fund I LP, which may include investments in health carerelated early-stage companies.

Reference information: / Clin Invest. 2019;129(3):955-957. https://doi.org/10.1172/JCI127100.

tration of the offending food allergen, the entire procedure requires close medical monitoring and, in some cases, rapid intervention with epinephrine.

\section{Targeting the source}

In this issue, Duan et al. (6) have taken a novel approach to improve the treatment of IgE-mediated anaphylaxis. The work depends on the constitutive expression of both the high-affinity receptor for IgE (FceRI) and CD33 (also known as Siglec-3) in mast cells. Unlike the FceRI, which in humans is highly expressed primarily in mast cells and basophils (7), CD33 is expressed on most myeloid cells, although the role of this receptor in cell signaling is not well understood (8). Duan et al. (6) generated liposomes bearing either the antigen trinitrophenol (TNP, TNP-LP), which would be recognized by anti-TNP $(\alpha$-TNP) IgE on the surface of mast cells, or TNP plus the previously described sialic acid analog CD33 ligand CD33L (TNPLP-CD33L). These liposomes were then extensively characterized using the human mast cell line LAD2 to demonstrate the specificity of the interaction with CD33.

Duan et al. (6) carefully demonstrated that delivery of TNP-LP-CD33L to mast cells could inhibit TNP-induced, FceRIdependent spleen tyrosine kinase (Syk) phosphorylation and downstream protein tyrosine kinase (PTK) phosphorylation, $\mathrm{Ca}^{++}$flux, and $\beta$-hexosaminidase release. The inhibition of this activation pathway only occurred if both the antigen (TNP, which reacts with TNP-IgE) and CD33L were on the same liposome (Figure 1). Moreover, pretreatment of LAD2 cells with TNP-LP-CD33L desensitized the human mast cells to subsequent antigen exposure. Duan and colleagues (6) also generated mice with mast cell-specific expression of human CD33, and in these animals, administration of TNP-LP-CD33L suppressed mast cell degranulation and cytokine secretion. Similar inhibitory effects, which could be sustained for at least sev- 
A

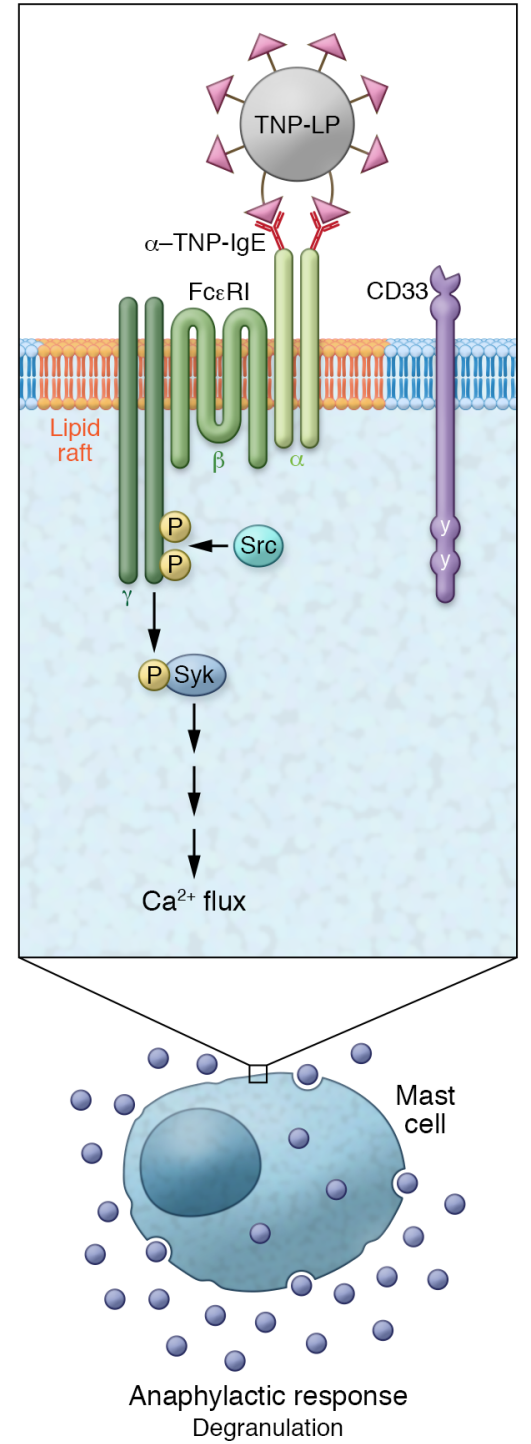

B

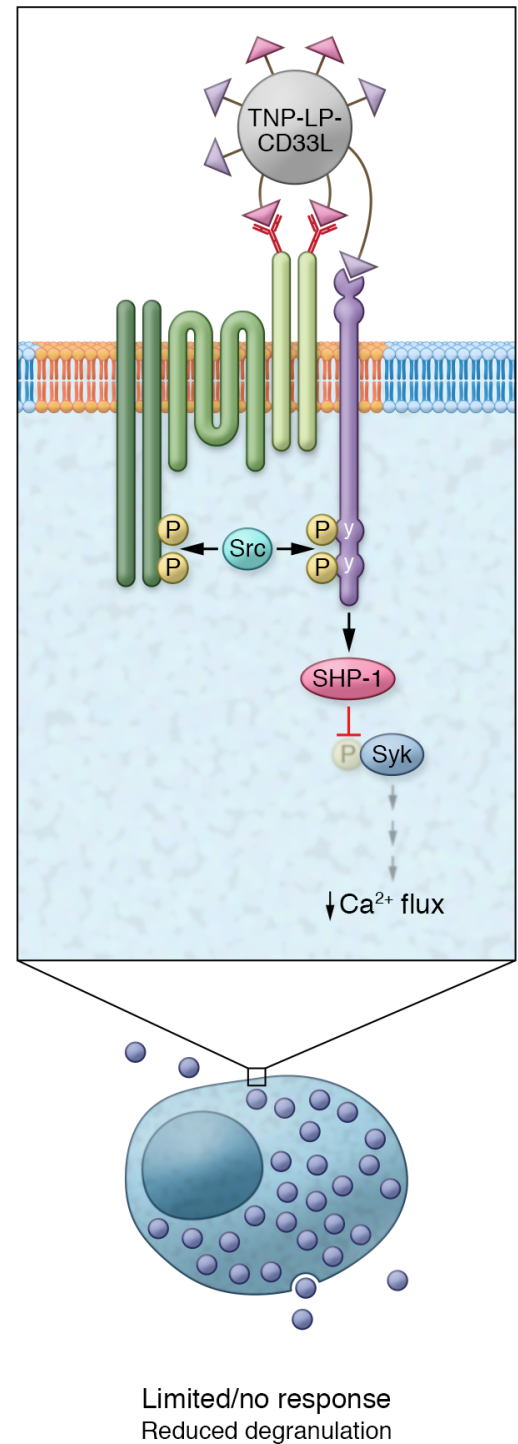

C

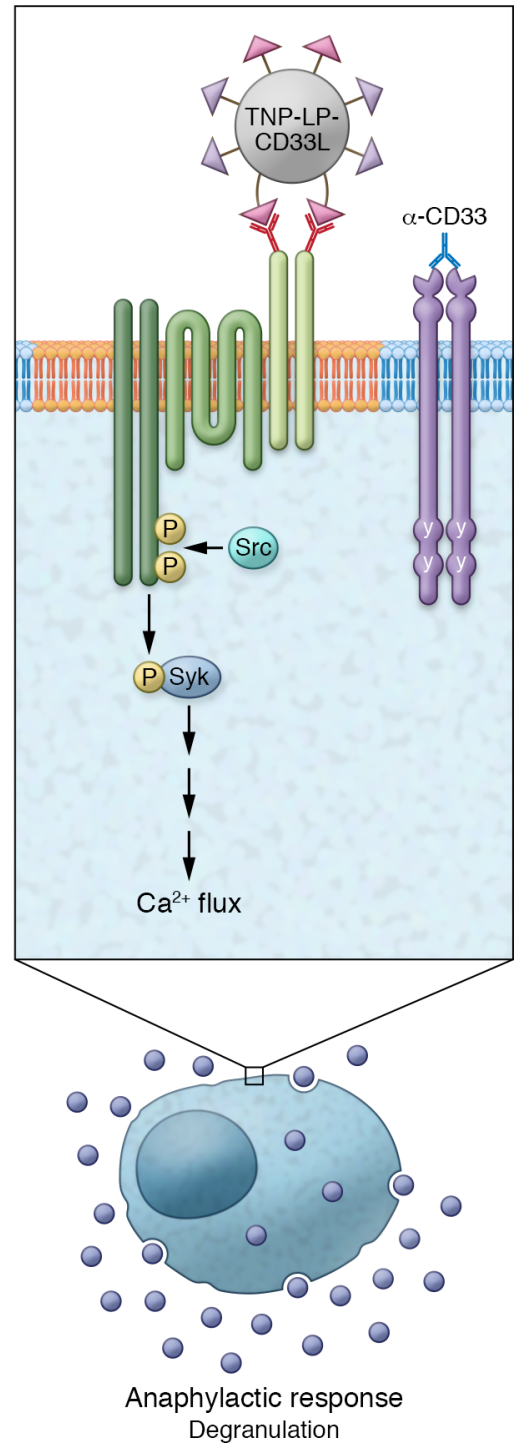

Figure 1. Recruitment of CD33 suppresses IgE/Fc\&RI signaling and mast cell activation. (A) TNP-LPs stabilize the $\alpha$-TNP-IgE-Fc\&RI complex in lipid rafts with Src kinases that initiate the Fc\&RI signaling cascade. Activation of this cascade results in extensive mast cell degranulation and mediator release. Duan et al. (6) suggest that CD33 has no detectable basal impact on Fc\&RI signaling, because it is not constitutively localized in the same microdomain as Fc $\varepsilon R I$, which localizes to lipid rafts. (B) TNP-LP-CD33L recruits CD33 to the $\alpha-T N P-I g E-F c \varepsilon R I$ immunological synapse. Duan and colleagues (6) suggest that the cytoplasmic ITIMs of CD33 are phosphorylated by Src kinases and then recruit the tyrosine phosphatase SHP-1, which dephosphorylates Syk and other kinases, thereby dampening the Fc\&RI-mediated signaling cascade. This results in a marked reduction in mast cell degranulation and mediator release. (C) Monoclonal $\alpha$-CD33 antibodies are proposed to block recruitment of CD33 to the lgE-FcERI complex, thereby enabling mast cell degranulation and mediator release induced by TNP-LP-CD33L.

en days in mouse skin, were shown in two different in vivo models of IgE-mediated immediate hypersensitivity (6).

\section{Clinical implications}

Does this elegant approach represent an important new treatment for potentially fatal IgE-associated reactivity in humans? Importantly, Duan et al. (6) showed that mast cells isolated from human skin express CD33 and that TNP-LP-CD33L essentially failed to induce degranulation of human lung mast cells, as shown by the inability of TNP-LP-CD33L, unlike TNP-LP, to induce bronchoconstriction of $\alpha$-TNP IgE-sensitized human precision-cut lung slices. In contrast, the same human precision-cut lung specimens that exhibited nearly full inhibition of FceRI-induced bronchoconstriction when challenged with TNP-LP-CD33L produced normal bronchoconstriction responses when challenged by carbachol, which works independently of the FceRI. While the results shown were obtained using lung slices derived from only two donors, the findings in such freshly obtained human lung specimens clearly show that the approach of Duan et al. (6) may indeed work when tested clinically in patients.

Increasingly, patients with IgE-associated allergies can often have adverse responses to multiple allergens (2). The study by Duan et al. (6) mainly focuses on using liposomal nanoparticles bearing a single antigen; however, the authors also 
tested the efficacy of their approach for reducing antigen-dependent pathology in mice with sensitivity to two antigens. Mice sensitized with both $\alpha$-TNP IgE and $\alpha$-OVA IgE were treated with OVA-LPCD33L and then challenged 5.5 hours later with either OVA-LP or TNP-LP. Treatment with OVA-LP-CD33L or subsequent challenge with OVA-LP did not result in detectable anaphylaxis; however, TNPLP induced anaphylaxis in both the OVALP-CD33L-treated and untreated mice (6). These results provide evidence that the antigenic desensitization induced by CD33L-containing antigenic liposomes is indeed antigen specific. Duan et al. (6) also showed that liposomes that display both the offending allergen and CD33L can not only prevent the antigen-dependent activation of IgE-bearing mast cells but can also enhance the clearance of circulating $\alpha$-allergen IgE, thus preventing resensitization and thereby inhibiting mast celldependent anaphylaxis for several days.

\section{Remaining questions and future directions}

The study by Duan et al. (6) leaves several questions unanswered. For example, what is the reason for the rather sustained unresponsiveness of mast cells after exposure to TNP-LP-CD33L? Does this lack of mast cell responsiveness reflect endocytosis of IgE-FceRI or the blocking of antigen binding to the remaining $\alpha$-allergen IgE on the mast cell surface, or both? Likewise, as mast cells appear not to be depleted by treatment with antigenic liposomes, they presumably will be resensitized by antigen-specific IgE upon expression of newly synthesized FceRI. Duan and colleagues (6) acknowledge this issue and raise the important point that it will be essential to determine how long it will take for mast cell resensitization in immunized animals. Moreover, the authors raise the possibility that treatment with an $\alpha$-IgE, such as omalizumab (Xolair), might be useful in retarding resensitization of mast cells by newly synthesized IgE (6).

Finally, Duan and colleagues (6) note that other members of the Siglec family may also have the potential to modulate mast cell responses and that human mast cells express several other immunoreceptor tyrosine-based inhibitory motifcontaining (ITIM-containing) Siglecs, including Siglec-6, Siglec-7, Siglec-8, and Siglet-9, in addition to CD33. Furthermore, others have shown that antibody-dependent cross-linking of FceRI to Siglec-7 or Siglec-9 can suppress IgE-mediated mast cell degranulation $(9,10)$. It has also been shown, in the first report documenting an inhibitory effect of Siglec engagement on human mast cells, that antibody-mediated ligation of Siglec-8 could suppress FceRImediated $\mathrm{Ca}^{++}$flux and human mast cell degranulation (11). As stated by Duan et al., this evidence raises the possibility that "ligands for other Siglecs could substitute for CD33L in the antigenic liposomal nanoparticle platform for the suppression of mast cell-mediated anaphylaxis, making the family of Siglecs attractive targets for the development of therapeutics to treat allergies" (6). It remains to be seen whether new treatment strategies for allergen immunotherapy will indeed be based on the ability of CD33 or other similar molecules to desensitize tissue mast cells; however, the study by Duan et al. (6) is an excellent start in this direction.

\section{Acknowledgments}

The author's studies of mast cells and basophils are funded by the National Institute of Allergy and Infectious Diseases (NIAID), NIH (U19AI104209, to SJG, principal investigator [PI]); the United States-Israel Binational Science Foundation (2017182, to SJG and Ronit Sagi-Eisenberg, co-PIs); the National Institute of Arthritis and Musculoskeletal and Skin Diseases (NIAMS), NIH (R01 AR067145, to SJG, PI); the NIAID, NIH (R01 AI125567-01A1, to Scott Boyd, PI); the NIAID, NIH (R01 AI32494, to SJG, PI); and the Tobacco-Related Disease Research Program, University of California (SPO 127515, to SJG, PI).

Address correspondence to: Stephen J. Galli, Department of Pathology, Stanford University School of Medicine, Center for Clinical Sciences Research, 269 Campus Drive, Room 3255b, Stanford, California 94305-5176, USA. Phone: 650.736.6014; Email:sgalli@stanford.edu.

1. Wood RA, et al. Anaphylaxis in America: the prevalence and characteristics of anaphylaxis in the United States. J Allergy Clin Immunol. 2014;133(2):461-467.

2. NIAID-Sponsored Expert Panel, et al. Guidelines for the diagnosis and management of food allergy in the United States: report of the NIAID-sponsored expert panel. JAllergy Clin Immunol. 2010;126(6 suppl):S1-S58.

3. Sampson HA, et al. Symposium on the definition and management of anaphylaxis: summary report. J Allergy Clin Immunol. 2005;115(3):584-591.

4. Bauer RN, Manohar M, Singh AM, Jay DC, Nadeau KC. The future of biologics: applications for food allergy. J Allergy Clin Immunol. 2015;135(2):312-323.

5. Wood RA. Food allergen immunotherapy: Current status and prospects for the future. J Allergy Clin Immunol. 2016;137(4):973-982.

6. Duan S, et al. CD33 recruitment inhibits IgE-mediated anaphylaxis and desensitizes mast cells to allergen. J Clin Invest. 2019;129(3):1387-1401.

7. Galli SJ, Tsai M. IgE and mast cells in allergic disease. Nat Med. 2012;18(5):693-704.

8. Laszlo GS, Estey EH, Walter RB. The past and future of CD33 as therapeutic target in acute myeloid leukemia. Blood Rev. 2014;28(4):143-153.

9. Mizrahi S, Gibbs BF, Karra L, Ben-Zimra M, Levi-Schaffer F. Siglec-7 is an inhibitory receptor on human mast cells and basophils. J Allergy Clin Immunol. 2014;134(1):230-233.

10. Avril T, Floyd H, Lopez F, Vivier E, Crocker PR. The membrane-proximal immunoreceptor tyrosine-based inhibitory motif is critical for the inhibitory signaling mediated by Siglecs-7 and -9, CD33-related Siglecs expressed on human monocytes and NK cells. J Immunol. 2004;173(11):6841-6849.

11. Yokoi H, et al. Inhibition of FcepsilonRI-dependent mediator release and calcium flux from human mast cells by sialic acid-binding immunoglobulin-like lectin 8 engagement. J Allergy Clin Immunol. 2008;121(2):499-505.e1. 